\title{
Review: topical mupirocin or fusidic acid may be more effective than oral antibiotics for limited non-bullous impetigo
}

Koning S, Verhagen AP, van Suijlekom-Smit LW, et al. Interventions for impetigo. Cochrane Database Syst Rev 2004;(2):CD003261.

Which treatments are effective for impetigo?

\section{METHODS}

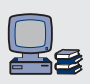

Data sources: Cochrane Skin Group Specialised Trials Register (March 2002), Cochrane Central Register of Controlled Trials

(Issue 1, 2002), National Research Register (2002), Medline (1966 to January 2003), EMBASE/Excerpta Medica (1980 to March 2000), LILACS (November 2001), and metaRegister of Controlled Trials on the Current Controlled Trials website; hand searches of Yearbook of Dermatology (1938-66) and Yearbook of Drug Therapy (1949-66); reference lists of retrieved articles; and pharmaceutical companies.

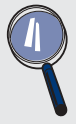

Study selection and assessment: published and unpublished randomised controlled trials (RCTs) in any language that assessed any intervention for impetigo (non-bullous, bullous, secondary, and impetiginised dermatoses) in patients with diagnosed impetigo or impetigo contagiosa, preferably confirmed by bacterial culture; studies that assessed patients with broadly defined bacterial skin infections or pyoderma were included if results for patients with impetigo were reported separately. 2 independent reviewers assessed the methodological quality of individual trials using the Jadad ( 3 items) and Delphi ( 9 items) scales.

Outcome: clinical cure or improvement (eg, clearance of crusts, blisters, and redness) assessed by investigators at 1 week after initiation of treatment.

\section{MAIN RESULTS}

57 trials (3533 evaluable patients) met the selection criteria. 38 different treatments (20 oral and 18 topical) were assessed. 12 of 57 trials were assessed as good quality (scores $\geqslant 50 \%$ on both quality scales).

Non-bullous impetigo. Topical antibiotics had better cure rates than placebo, oral antibiotics (specifically, mupirocin $v$ oral erythromycin), and disinfecting agents (table). No single topical antibiotic was superior to another. Oral antibiotics. Oral penicillin did not differ from placebo (l study). Several single studies compared different oral antibiotics, and significant differences in cure rates were found for the following: cefuroxim $v$ erythromycin ( 1 trial); erythromycin $v$ penicillin ( 2 trials); amoxicillin plus clavulanic acid $v$ amoxicillin alone ( 1 trial); and cloxacillin $v$ penicillin (2 trials). Disinfecting treatments (hexachlorophene) did not differ from placebo or oral antibiotics (penicillin) ( 1 trial).

Bullous impetigo. Topical antibiotics (neomycin/bacitracin or chloramphenicol) had lower cure rates than oral antibiotics (erythromycin) ( 1 trial). A comparison of different topical antibiotics found that

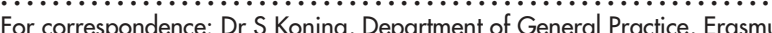
MC - University Medical Center Rotterdam, Rotterdam, The Netherlands. s.koning@erasmusmc.nl

Source of funding: not stated. fusidic acid had higher cure rates than neomycin/bacitracin or chloramphenicol ( 1 trial). A comparison of 2 oral antibiotics (cephalexin $v$ dicloxacillin) found no difference in cure rates.

\section{CONCLUSIONS}

Topical antibiotics (mupirocin or fusidic acid) may be slightly more effective than oral antibiotics (erythromycin) for patients with limited, non-bullous impetigo. Limited evidence suggests that disinfecting treatments are not effective.

A modified version of this abstract also appears in Evidence-Based Medicine.

Commentary

reatment of impetigo has a weak evidence base, and the review by Koning et al attempts to define what is known. Scant information is available on the natural course of impetigo, and limited practice guidelines have resulted in conflicting treatment recommendations. A review of the US Agency for Health Care Policy and Research database reveals no North American guidelines for treating impetigo. Current guidelines from standard textbooks ${ }^{1} 2$ indicate that treatment choices should be based on differentiation of the common staphylococcal variety of impetigo from streptococcal forms, extent and location of lesions, and accessibility of lesions to topical medication. Additional research is needed to identify appropriate treatment protocols for impetigo, including management of the possible development of antibiotic resistance.

The findings of this review are relevant to nurses working with children in outpatient clinics, paediatrics, and public health settings, as well as primary care nurse practitioners and community/clinical nurse specialists. They clearly show the existing variation and lack of consensus regarding the treatment of impetigo. The authors wisely caution that the use of topical antibiotics may lead to antibacterial resistance, which is an important consideration when selecting a treatment regimen.

The findings provide guidance to clinicians in treating non-bullous impetigo. Topical antibiotics such as mupirocin and fusidic acid appear to be more effective than oral antibiotics and disinfecting agents for limited disease, although equivalence cannot yet be ruled out. Because the natural course of impetigo is not well understood, treatment and future research should focus not only on curing the condition but also on preventing its spread.

D Shane Strickland, RN, MScN (PHCNP cert) Lakehead University School of Nursing Thunder Bay, Ontario, Canada

1 Hoekelman RA, Adam HM, Nelson NM, et al, editors. Primary pediatric care. Fourth edition. Philadelphia: Mosby, 2001:1343-5.

2 Burns CE, Dunn AM, Brady MA, et al. Pediatric primary care. Third edition. Philadelphia: WB Saunders, 2004:1070-2.

Topical antibiotics $v$ various treatments for clinical cure (or improvement) of non-bullous impetigo at 1 week ${ }^{*}$

\begin{tabular}{lclcl}
\hline Comparison & Number of trials $(\mathbf{n})$ & Weighted event rates & RBI (95\% Cl) & NNT (CI) \\
\hline Topical antibiotics $v$ placebo & $5(365)$ & $58 \%$ v $21 \%$ & $179 \%$ (108 to 274) & $3(3$ to 4$)$ \\
Topical mupirocin $v$ oral erythromycin & $10(581)$ & $91 \% \vee 85 \%$ & $7 \%(1$ to 13$)$ & 17 (9) 100$)$ \\
Topical antibiotics $v$ disinfecting treatment & $2(292)$ & $72 \% \vee 62 \%$ & $15 \%(1$ to 32$)$ & Borderline significance \\
\hline
\end{tabular}

*Abbreviations defined in glossary; RBI, NNT, and Cl calculated from data in article. 